\title{
Protracted Cardiovascular Impairments After Anterior Cruciate Ligament Injury: A Critically Appraised Topic
}

\author{
Cody R. Butler, Kirsten Allen, Lindsay J. DiStefano, and Lindsey K. Lepley
}

\begin{abstract}
Clinical Scenario: Anterior cruciate ligament (ACL) tear is a devastating knee injury with negative long-term consequences, such as early-onset knee osteoarthritis, biomechanical compensations, and reduced physical activity. Significant reduction in physical activity is a powerful indicator of cardiovascular (CV) disease; therefore, those with a history of ACL injury may be at increased risk for CV disease compared with noninjured individuals. Focused Clinical Question: Do individuals with a history of ACL injury demonstrate negative CV changes compared with those without a history of ACL injury? Summary of Key Findings: Three articles met the inclusion criteria and investigated CV changes after ACL injury. Both cross-sectional studies compared participants with ACL injury with matched controls. Bell et al compared time spent in moderate to vigorous physical activity and step count, whereas Almeida et al compared maximum rate of oxygen consumption, ventilatory thresholds, isokinetic quadriceps strength, and body composition. Collectively, both quantitative studies found that individuals with a history of ACL injury had less efficient CV systems compared with matched controls and/or preoperative data. Finally, a qualitative study of 3506 retired National Football League athletes showed an increased rate of arthritis and knee replacement surgery after an ACL injury when compared with other retired National Football League members, in addition to a $>50 \%$ increased rate of myocardial infarction. Clinical Bottom Line: A history of ACL injury is a source of impaired physical activity. Preliminary data indicate that these physical activity limitations negatively impair the CV system, and individuals with a history of ACL injury demonstrate lower maximum oxygen consumption, self-reported disability, and daily step count compared with noninjured peers. These complications support the need for greater emphasis on CV wellness. Strength of Recommendation: Consistent findings from 2 cross-sectional studies and 1 survey study suggest level IIB evidence to support that ACL injury is associated with negative $\mathrm{CV}$ health.
\end{abstract}

Keywords: cardiovascular diseases, coronary artery heart disease, exercise, rehabilitation and therapy, rehabilitation

\section{Clinical Scenario}

Traumatic knee injuries lead to a multitude of negative effects on the body, including altered physical activity, biomechanical compensations, and early onset knee osteoarthritis. ${ }^{1-5}$ Individuals recovering from anterior cruciate ligament (ACL) injury spend less time in moderate to vigorous physical activity and take fewer steps per day, compared with healthy individuals. ${ }^{1}$ Significant reductions in physical activity are a powerful indicator of cardiovascular disease. Accordingly, those with a history of ACL injury may be at an increased risk of cardiovascular disease compared with their nonsurgical peers.

\section{Focused Clinical Question}

Do individuals with a history of an ACL injury demonstrate negative cardiovascular changes compared with those who have not experienced an ACL injury?

\section{Summary of Search, "Best Evidence" Appraised, and Key Findings}

- A literature search was performed to investigate cardiovascular changes after ACL injury.

The authors are with the University of Connecticut, Storrs, CT. Butler (cody.butler@ uconn.edu) is corresponding author.
- All studies must have investigated the protracted cardiovascular changes (such as heart rate, blood pressure, and oxygen consumption) following ACL injury.

- Of the initial 106 articles retrieved, 3 met the inclusion criteria, 2 cross-sectional studies, and 1 qualitative survey.

- Cardiovascular testing ranged from presurgery to 6 months postsurgery, ${ }^{2}$ as well as multiple years postsurgery. ${ }^{1}$

- Both cross-sectional studies ${ }^{1,2}$ compared ACL reconstruction (ACLR) participants with matched healthy controls. One study used accelerometers and measured time spent in moderate to vigorous physical activity and step count between groups (ACLR vs controls) for 7 days. The other cross-sectional investigation compared maximum rate of oxygen consumption $\left(\mathrm{VO}_{2} \max \right)$, ventilatory thresholds, isokinetic strength, and body composition between groups. It examined participants with ACL injury at presurgery and 6 months postoperation. Results were also compared with healthy controls.

- The qualitative survey study ${ }^{3}$ showed an increased rate of arthritis and knee replacement surgery after an ACL injury. In addition, although statistical significance was not reached, there was a $>50 \%$ increased rate of myocardial infarction in players with a history of ACL tear.

- Although 1 study found that participants with a history of ACL injury had better cardiovascular outcomes compared with presurgical data, ${ }^{2}$ collectively, both cross-sectional studies found that individuals with a history of ACL injury had less efficient cardiovascular systems compared with healthy controls. 
- Deficits in oxygen consumption, heart rate, and self-reported function associated with cardiac events were observed in the ACL-injured groups.

\section{Clinical Bottom Line}

A history of an ACL injury and reconstruction significantly influences physical activity. For instance, those with a history of ACL injury reportedly have a lower daily step count and decreased aerobic fitness. ${ }^{1}$ These significant declines in physical activity may negatively impair the cardiovascular system. Strikingly, a recent qualitative report concluded that those with a history of ACLR reportedly have a $>50 \%$ increased risk of myocardial infarction. ${ }^{3}$ This risk of cardiovascular disease, taking into account the wellestablished risk of early onset osteoarthritis complications that plague this population, may indicate that individuals with a history of ACL injury that develop early onset osteoarthritis are at an increased risk of cardiovascular disease. ${ }^{3}$ Though these are early data, this link between osteoarthritis and cardiovascular disease has been found in idiopathic osteoarthritis development. ${ }^{6,7}$ Altogether, this clinical scenario suggests that there is a risk of cardiovascular complications after ACL injury, and there is a need to consider incorporating cardiovascular-focused rehabilitation strategies.

\section{Strength of Recommendation}

Consistent preliminary findings from 2 cross-sectional studies and 1 survey study suggest that level IIB evidence to support that ACL injury is associated with negative cardiovascular health.

\section{Search Strategy}

\section{Terms Used to Guide Search Strategy}

- Participant/Client group: individuals who have undergone knee surgery

- Intervention/Assessment: cardiovascular function $\left(\mathrm{VO}_{2} \mathrm{max}\right.$, blood pressure $[\mathrm{BP}]$, heart rate $[\mathrm{HR}]$, heart rate variability [HRV], and cardiovascular disease)

- Comparison: nonsurgical

- Outcome: aerobic fitness or cardiovascular function/disease

\section{Sources of Evidence Searched (Databases)}

- PubMed

- SPORTDiscus

\section{Inclusion and Exclusion Criteria}

\section{Inclusion}

- Human participants only

- Articles available in the English language

- Group of postknee orthopedic surgical participants

- Measure of cardiovascular health ( $\mathrm{VO}_{2} \max , \mathrm{BP}, \mathrm{HR}, \mathrm{HRV}$, and cardiovascular disease)

\section{Exclusion}

- Animal participants

- Languages other than English

- Participants with other major lower-extremity injury

\section{Results of Search}

Three relevant studies were located and categorized and are presented in Table 1.

The following studies were identified as the "best" evidence and selected for inclusion in the critically appraised topic. Reasons for selecting these studies were as follows:

- All studies matched the inclusion/exclusion criteria.

- All studies contain groups with a knee-specific orthopedic surgery.

- All studies utilized cardiovascular-based outcomes.

\section{Implications for Practice, Education, and Future Research}

The 2 cross-sectional studies included in this critically appraised topic found a significant decrease in cardiovascular measures when compared with healthy controls. ${ }^{1,2}$ Both studies compared the results of athletes (aged 20-22 y) who had torn their ACL and underwent reconstructive surgery versus a healthy matched control. Bell et $\mathrm{al}^{1}$ found that individuals with a history of ACL injury spent less time in moderate to vigorous physical activity and had lower daily step counts, which are variables that have a direct effect on cardiovascular health. Almeida et $\mathrm{al}^{2}$ evaluated the cardiovascular system via $\mathrm{VO}_{2}$ max and ventilatory thresholds, as well as a knee function questionnaire, isokinetic strength test, and body composition measurement. This study found that participants with a history of ACL injury scored significantly lower in all categories. Cumulatively, these results indicate that those with a history of ACL injury may be at risk for a compromised cardiovascular system compared with their nonsurgical peers. ${ }^{1,2}$ Finally, the qualitative study showed that out of 3506 former National Football League athletes, those who had torn their ACL had a higher incidence of myocardial infarction compared with other retired National Football League members. ${ }^{3}$ In brief, there is a short-term, immediate problem demonstrated by the 2 cross-sectional studies reporting significant reductions in physical activity; and, long-term, there is a troubling qualitative study that points to a high incidence of cardiac events. See Table 2 for article characteristics.

On a global scale, ACL ruptures occur in 300,000 Americans every year, ${ }^{8}$ with a reported reinjury rate as high as $23 \%,{ }^{9}$ which may also influence declines in physical activity rates. These statistics, in conjunction with the findings of this critically appraised topic, and the link between osteoarthritis and cardiovascular disease in idiopathic populations, ${ }^{6,7}$ demonstrate the necessity of further studies to better determine the long-term impairments of ACL injury.

Although there is extensive literature studying the long-term changes of ACL injury on the knee joint, ${ }^{10}$ the evidence regarding the long-term cardiovascular changes after knee surgery is limited,

\section{Table 1 Summary of Study Design of Articles Retrieved}

\begin{tabular}{lll}
\hline Level of evidence $^{\mathbf{a}}$ & Study design & Reference \\
\hline 3 & Cross-sectional study & Bell et al \\
3 & Cross-sectional study & Almeida et $\mathrm{al}^{2}$ \\
6 & Survey study & Meehan et $\mathrm{al}^{3}$ \\
\hline
\end{tabular}

${ }^{\mathrm{a} O x f o r d}$ Centre for Evidence-Based Medicine Best Evidence. 


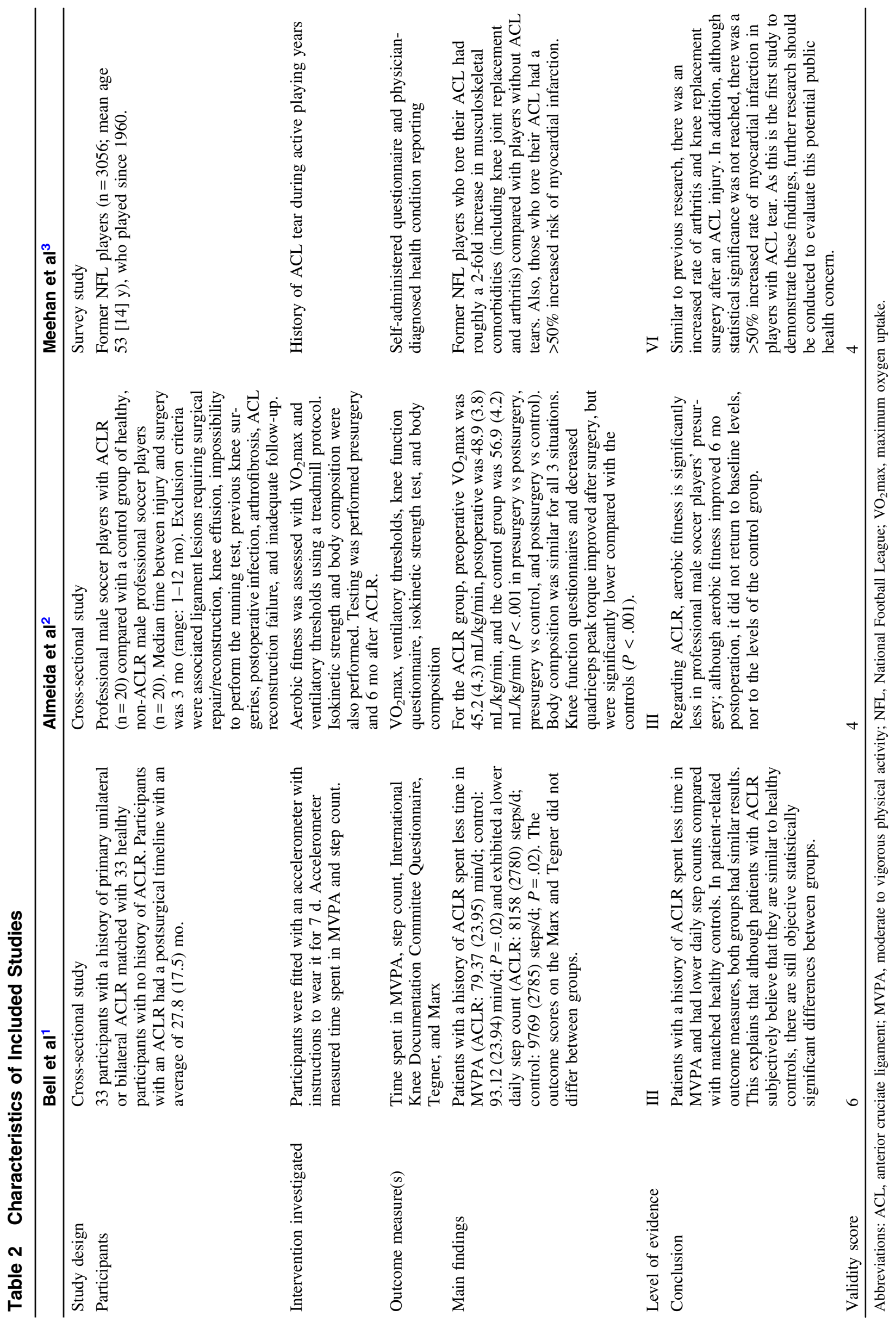


and, as such, future research is needed to better understand the early signs of cardiovascular distress and how it evolves over time. A refined understanding of the timeline of cardiovascular changes would help both the researcher and clinician better understand how to treat these impairments transcribed from ACL injury. It is important to note that of the 3 appraised articles, only one article assessed cardiovascular fitness presurgery. ${ }^{1}$ Future research should focus on the effects of ACL injury compared with ACL reconstruction.

Current trends of ACL rehabilitation focus on regaining strength and functional movements for return to play goals. Clinicians tend to tailor therapy to patient-centered goals, such as being able to run, play a particular sport, or successfully perform activities of daily living. Unfortunately, $88 \%$ of patients expect to return to sport ${ }^{11}$ despite alarmingly high reinjury rates. ${ }^{9}$ Plausibly, this decline in preinjury level of sport could be an important link to cardiovascular disease later in life. Future research will need to evaluate this relationship. The results from this topic indicate a need for greater focus on cardiovascular-based therapy during rehabilitation and encouragement to return to endurance-based physical activity postrehabilitation. The evidence presented should also encourage clinicians to modify current trends of ACL rehabilitation strategies to also focus on cardiovascular health after ACL injury.

As the data to observe progressive cardiovascular changes are not yet available, longitudinal studies will be beneficial to understand the relationship between ACL injury and long-term cardiovascular health. The emerging picture, at the very least, suggests that cardiovascular fitness should be an important component of rehabilitation, which should be on a clinician's radar.

\section{References}

1. Bell DR, Pfeiffer KA, Cadmus-Bertram LA, et al. Objectively measured physical activity in patients after anterior cruciate ligament reconstruction. Am J Sports Med. 2017;45(8):1893-1900. PubMed ID: 28419817 doi:10.1177/0363546517698940

2. Almeida AM, Santos Silva PR, Pedrinelli A, Hernandez AJ. Aerobic fitness in professional soccer players after anterior cruciate ligament reconstruction. PLoS One. 2018;13(3):e0194432. PubMed ID: 29566090 doi:10.1371/journal.pone.0194432

3. Meehan WP, Weisskopf MG, Krishnan S. Relation of anterior cruciate ligament tears to potential chronic cardiovascular diseases. Am J Cardiol. 2018;122(11):1879-1884. PubMed ID: 30282598 doi:10.1016/j.amjcard.2018.08.030

4. Golightly YM, Marshall SW, Callahan LF, Guskiewics K. Earlyonset arthritis in retired National Football League players. J Phys Act Health. 2009;6:638-643. PubMed ID: 19953841 doi:10.1123/jpah.6. 5.638

5. Moretz JA III, Harlan SD, Goodrich J, Walters R. Long-term follow-up of knee injuries in high school football players. Am J Sports Med. 1984;12:298-300. PubMed ID: 6476189 doi:10.1177/ 036354658401200410

6. Rahman MM, Kopec JA, Anis AH, Cibere J, Goldsmith CH. Risk of cardiovascular disease in patients with osteoarthritis: a prospective longitudinal study. Arthritis Care Res. 2013;65:1951-1958. doi:10. 1002/acr.22092

7. Grindulis KA, Bhatia G, Davis R, Sosin M, Connolly D, Khattak F. Osteoarthritis and cardiovascular death. Ann Rheum Dis. 2003; 62:495. PubMed ID: 12695176 doi:10.1136/ard.62.5.495-b

8. Griffin LY, Albohm MJ, Arendt EA, et al. Understanding and preventing noncontact anterior cruciate ligament injuries. Am J Sports Med. 2006;34:1512-1532. PubMed ID: 16905673 doi:10.1177/ 0363546506286866

9. Shelbourne KD, Gray T, Haro M. Incidence of subsequent injury to either knee within 5 years after anterior cruciate ligament reconstruction with patellar tendon autograft. Am J Sports Med. 2009;37(2):246-251. PubMed ID: 19109531 doi:10.1177/ 0363546508325665

10. Lie MM, Risberg MA, Storheim K, Engebretsen L, Øiestad BE. What's the rate of knee osteoarthritis 10 years after anterior cruciate ligament injury? An updated systematic review. Br J Sports Med. 2019;53(18):1162-1167. PubMed ID: 30936063 doi:10.1136/ bjsports-2018-099751

11. Webster KE, Feller JA. Expectations for return to preinjury sport before and after anterior cruciate ligament reconstruction. Am J Sports Med. 2019;47(3):578-583. PubMed ID: 30649903 doi:10.1177/ 0363546518819454 